\title{
A Conceptual Framework for Chronic Disease Prevention Based on Multilevel Approach
}

\author{
Fadumo Abdi Noor ${ }^{1}$, Gabriel Gulis ${ }^{1} \&$ Jens Søndergaard ${ }^{2}$ \\ ${ }^{1}$ Unit for Health Promotion Research, Department of Public Health, University of Southern Denmark, Niels \\ Bohrsvej 9-10, Esbjerg, Denmark \\ ${ }^{2}$ Research Unit for General Practice, Department of Public Health, University of Southern Denmark, J.B. \\ Winslows Vej 9A, 5000 Odense, Denmark \\ Correspondence: Fadumo Abdi Noor, Unit for Health Promotion Research, University of Southern Denmark, \\ Niels Bohrsvej 9-10, 6700 Esbjerg, Denmark. Tel: 45-65-508-692. E-mail: fanoor@health.sdu.dk
}

Received: January 19, 2018 Accepted: April 9, 2018 Published online: April 19, 2018

doi:10.5539/gjhs.v10n5p175 URL: https://doi.org/10.5539/gjhs.v10n5p175

\begin{abstract}
Aim: The aim of this paper is to present a conceptual framework for the analysis of chronic disease prevention work according to the principles of a multilevel approach to T2DM in Denmark.

Method: We conducted a review of published papers using PubMed, EMBASE, Web of Science, Google, Google Scholar, NICE Evidence Search, and we extended our search to include grey zone literature. We chose to focus our literature review on the collaboration between the different actors in the health system and the prevention and management of type 2 diabetes. We reviewed abstracts, and our search yielded a final total of 52 papers, of which we retained 18 and eliminated papers which were not related explicitly to the subject.
\end{abstract}

Results: Results showed that prevention and management of type T2DM must address multiple factors at multiple levels (intrapersonal, interpersonal and structural level) and within multiple settings (medical settings, communities/municipalities, regions and government). To analyze chronic disease prevention from a multilevel approach perspective, a conceptual framework was developed, which would guide the analytical process. Interventions should tackle specific risk factors within specific populations and by different actors who need to act in a coordinated manner.

Conclusion: It is becoming increasingly clear that a multilevel approach is needed to prevent chronic diseases. Working at multiple levels with multiple actors in the health system will hopefully help fight the increasing numbers of chronic diseases.

Keywords: collaboration, Denmark, diabetes prevention, health promotion, multilevel approach

\section{Introduction}

Chronic diseases are the leading causes of mortality and morbidity and are likely to impose an even larger burden in the future in Denmark (Carstensen, Kristensen, Ottosen, \& Register, 2008; Guariguata, Whiting, Hambleton, \& Beagley, 2013). Effective interventions involve a multidisciplinary and multilevel approach: multilevel approach is defined as "models in sociology attempting to identify the effects of social context on individual level outcomes" ("multi-level models - Dictionary definition of multi-level models | Encyclopedia," n.d.). Applied to our study, the definition of multilevel approach is to model effects of interactions of micro, meso and macro levels (as described later) on diabetes prevalence in the population.

International research shows that health systems can be designed to prevent and manage chronic diseases more effectively by applying a multilevel approach, but it is essential that a system level change is accompanied by a supportive environment, and the empowerment and active participation of individuals, families, communities and the government (Paquette-Warren, Tompkins, \& Harris, n.d.; Whittemore et al., 2016). A multilevel understanding of health signals the importance of the inter-relationship between the environment and the individual person.

This review is based on Cook's multilevel approach categorization (Cook, Purdie-Vaughns, Meyer, \& Busch, 2014). Using diabetes as an example of a chronic disease, it aims to identify what kinds of prevention measures are relevant on different levels and how the different levels cooperate: 
- Micro level - intrapersonal level, individuals and the dynamics which affect people's experiences with health and environment (family, peer groups, neighbourhood, etc.)

- Meso level - interpersonal, environmental level, interactions of different groups within specified environments such as municipality, and primary health care

- Macro level - structural level, social-political environment, policies, laws, and regulations

At the micro level (intrapersonal level), it is the personnel who execute the actual preventative work. This may be a physician, nurse, dietician, physiotherapist or prevention manager. The responsibility to detect a disease early lies with professionals in both general practices, in municipalities, in hospitals and also among citizens themselves. A proactive response from the health system may lead to diagnosis at an early stage; thereby complications of the disease can be prevented (Schwarz, Schwarz, Schuppenies, Stefan, \& Schulze, 2016).

The meso level (interpersonal level), which is the level of operative primary health care, may be used by health professionals responsible for activities related to diabetes prevention in municipalities, health districts, health care centres, workplaces, the private sector or local level non-governmental organizations. In Denmark, the municipalities contribute to early detection as part of the citizen-oriented prevention of diabetes. In line with the Health in All Policies principles ("Health in All Policies (HiAP) framework for country action," 2014), the municipalities may also include non-health professionals in early detection. Their actions are significant in relation to health promotion and primary prevention of lifestyle diseases, in sense of planning and timely conduct of activities in municipalities to influence prevalence of the disease (Lerouge, $\mathrm{Ph}$, Gaynor, \& $\mathrm{Ph}, 2010$ ).

At the macro level (structural level), the key issue is the sustainability of health systems. According to the Dahlgren \& Whitehead model of determinants of health, the upper level of general socioeconomic, cultural and environmental conditions represents the macro level (Whitehead, Dahlgren, \& Gilson, 2009). National health policies are expected to set the frameworks for actions on every level. Within these, it is the national level decision-makers' role to generate the prerequisites for diabetes prevention

Focus on programs which increase access to health promotion and disease prevention activities, early diagnoses, diabetes care and treatment and promote public awareness about diabetes through various methods, such as diabetes education and self-management practices, is needed

The aim of this paper is to present a conceptual framework developed for the analysis of chronic disease prevention work according to the principles of multilevel approach. To operationalize the theoretical framework, we selected type 2 diabetes mellitus (T2DM) as an example of a chronic disease. T2DM is a disease with large population health impact, existing broad knowledge of key risk factors and determinants of health leading to the disease and therefore offers good possibilities for prevention actions on any level of multilevel approach. Yet, our aim was to develop a conceptual framework applicable to any chronic disease; therefore T2DM as such is not discussed in detail in this article.

\section{Method}

A narrative review of literature was performed to identify application of multilevel approach on prevention of T2DM. The following electronic databases were searched: PubMed, EMBASE, Web of Science, Google, Google Scholar, NICE Evidence Search. The literature search strategy included terms like 'management of type 2 diabetes', 'health promotion', 'multilevel approaches', 'risk factors', 'health policy (Mesh)', 'health services administration (Mesh)', 'intersectoral collaboration' (Mesh) combined with 'type 2 diabetes' and 'prevention'. The search strategy also involved a search of reference lists of literature on the topic. During the period of time from 1997-2017, we searched literature published in English and Danish. In addition, the NICE guidance (National Institute of Healthcare and Excellence, 2011) was further searched to identify detailed recommendations about diabetes prevention, risk factors, and what should be targets for lifestyle change. A set of exclusion and inclusion criteria was used to identify as relevant and current papers as possible for the review. Exclusion criteria were articles not relevant to the topic under investigation, not written in English or Danish, dated prior to 1997, articles and reports focusing only on the clinical part of collaboration, and articles and reports not focusing on the multilevel approach and T2DM. Papers from areas such as primary care and community care collaboration and type 2 diabetes were included, as well as articles and reports from countries outside Denmark. 


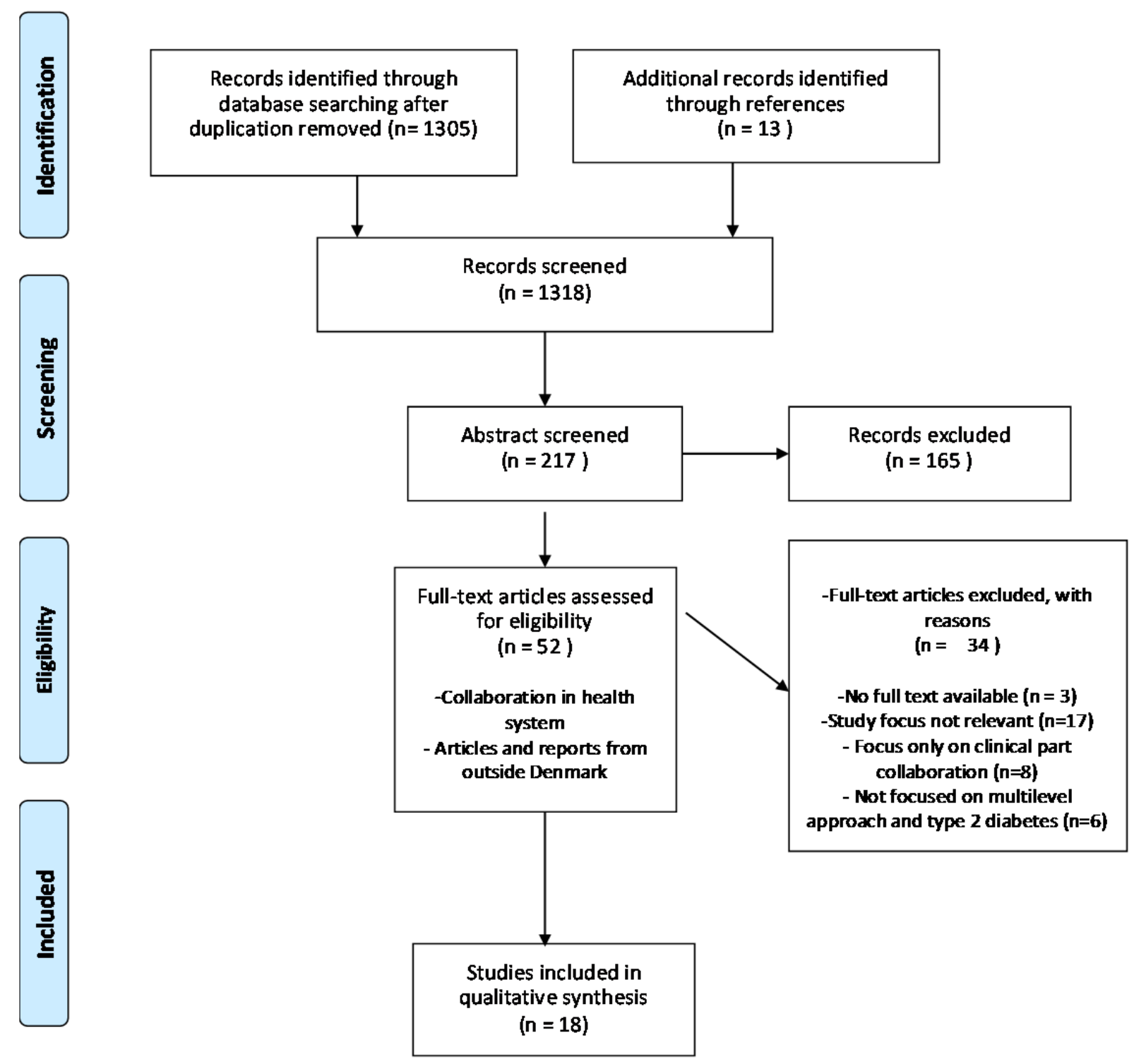

Figure 1. Flow chart of the literature search process

\section{Results}

In the review, a number of 18 studies were found relevant based on the multilevel approach and T2DM. In general the studies show that prevention of type T2DM must address multiple factors at multiple levels within multiple settings (medical settings, communities/municipalities, regions and government)(Paquette-Warren et al., n.d.; Whittemore et al., 2016). To analyse chronic disease prevention from a multilevel approach perspective therefore requires the development of a conceptual framework, which would guide the analytical process.

Few studies describe what kind of activities should be undertaken at micro level and by whom $(14,15)$. The key actors at micro level are the general practitioners (GPs), and interventions should tackle individual risk factors, behaviour change, and follow-up. Such an individual approach focuses on high-risk or affected individuals. The primary prevention tasks of general practice are the identification of risk factors, early detection, and the assessment of whether the patient may benefit from prevention interventions. General practice should act as a coordinator for the patient with T2DM, the treatment and preventions of both disease-specific symptoms and symptoms of a more general nature thus ensuring the consistency of measures. It must be aware of the patient's lifestyle and assess the need for preventive services with the aim of reducing the risk of disease, support the patient's motivation for lifestyle changes and offer to refer to services in the municipality and/or undertake specific lifestyle intervention in practice if the patient can benefit from it (Ist \& Sanit, 2015; Zaletel, Piletic, Lindström, 
Icks, \& Rothe, 2015).

According to the literature (Paquette-Warren et al., n.d.; Schwarz et al., 2016; Sørensen, Maggini, \& Kuske, 2015) at meso level, the key actions should aim at hospital-based care and community-based health promotion / disease prevention intervention and should be conducted by health care workers at hospitals; doctors, nurses, biomedical scientist etc., as well as health workers in the municipality; dieticians, physiotherapists or prevention managers. The results also showed that (Ansari, Dixon, \& Browning, 2013), at the meso level, the municipality's/community's responsibility is to increase awareness about diabetes and other CVD, and related risk factors; educational, motivational and skill programming for increasing positive health behaviours by including citizens/patients and their families. This is done by offering community-based activities and interventions focusing on physical activity, weight, and diet over a course of time, and motivational meetings are also provided for the patients/citizens enabling the patients to express their thoughts and expectations with the aim of encouraging and guiding them to participate in the different activities provided by the municipality (Sørensen et al., 2015).

The prevention tasks for the hospital are to integrate prevention into a treatment process and to support a health-promoting environment. Based on the patients' needs, the hospital staffs refer the patients to preventive actions and support the patients' motivation for lifestyle changes. For hospitalized patients, the hospital integrates appropriate preventive measures related to the treatment provided under the admission. Hospitals and municipalities have a joint responsibility to ensure quality standards and coherent patient education.

At macro level, key interventions are expected to address the structural level with impact on a larger population (Paquette-Warren et al., n.d.). Funding structures, reform strategies, taxation, and local/regional/ national support structures are the main tools. The macro level is characterized by leadership commitment to a chronic disease (national diabetes plan), management approach and improved quality of services, and the availability of necessary resources such as staffing and medical supplies. Actors at this level are policy makers both at national and regional level.

Identifying strategies addressing healthy public policies was reported to have an important impact on high-risk individuals and to prevent the onset of diabetes in those at risk. Findings also showed the significance of a macro level addressing environmental interventions aimed at minimizing exposure to and reducing risk factors for diabetes, particularly healthy food and physical activity (Golden, Mcleroy, Green, Earp, \& Lieberman, 2015; Green, Brancati, \& Albright, 2012). The purpose of the population-based interventions is to integrate and link evidence-based activities which are planned and coordinated nationally and implemented on national, regional and local levels. Engaging in or at least taking account of the impact of all sections and levels of society when planning and implementing diabetes plans are fundamental to achieving a great outcome in the prevention of T2DM (Lewis, Fitzgerald, Zulkiewicz, Peinado, \& Williams, 2016; Zaletel et al., 2015).

Several studies and reports concluded that (Fitzgerald et al., 2016; Green et al., 2012; Whittemore et al., 2016) the collaboration between the different levels includes better organization of health information and its timely availability, a reduction in duplication of services, and better health system planning. Furthermore, it encompasses adherence to evidence-based practice guidelines, principles related to patient-centred care, proactive actions and population-level health promotion/disease prevention actions (Paquette-Warren, Tyler, Fournie, \& Harris, 2017).

The conceptual framework to guide the analysis of T2DM management according to a multilevel approach in Denmark is presented in Figure 2. 


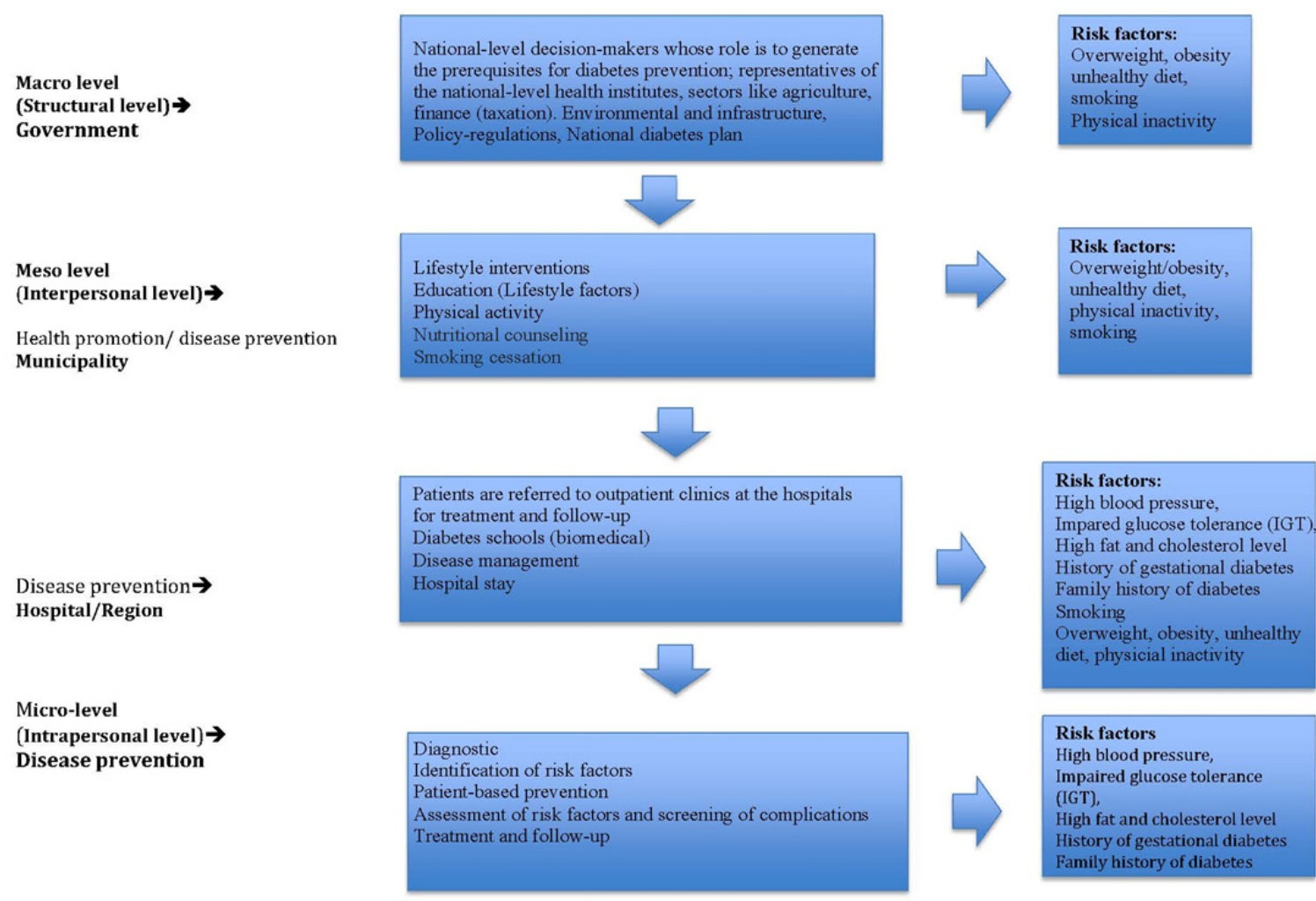

Figure 2. Conceptual framework to illustrate the application of multilevel approach in the prevention of T2DM in Denmark

\section{Discussion}

The proposed analytical framework confirms the need for use of coordinated and harmonized prevention strategies assigned to key actors at different levels according to the multilevel approach.

There are two key issues to discuss with regard to the subject of our work: what is the alternative to multilevel approach and the content of the conceptual framework. Regarding the alternatives, until last decades, health systems traditionally focused on a rather individualistic approach with a narrow approach having the disease in the centre. Modern public health introduced the issues of complexity and system science (de Montigny, Desjardins, \& Bouchard, 2017; Rutter et al., 2017; Salway \& Green, 2017)fully justifying the multilevel approach. In fact, there is no alternative to such approach! As it appears from the T2DM example shown, prevention requires the involvement of several professional disciplines, including medicine, behavioural science, nutrition, and physical activity. Clinical studies have consistently shown that diabetes can be prevented by lifestyle changes related to healthy nutrition, and an adequate amount of physical exercise and weight reduction (Deshpande, Dodson, Gorman, \& Brownson, 2008). To build a strong prevention team and network of professionals, the aim is to engage experts from various backgrounds who have an interest in reducing the growing prevalence of T2DM and to strengthen the community action.

In terms of content of the conceptual framework, it can be agreed that it provides both an analytical tool as expected as well as, more importantly, a planning tool. It allows identifying key stakeholders both on individual and institutional levels. For example, professionals (of different backgrounds) should be aware whether individuals are at risk of developing type T2DM and develop collaboration amongst them. A municipality, as a representative of a community, is responsible for creating an intervention framework to encourage and support healthy lifestyle choices aimed at changing the knowledge, attitudes and beliefs of the citizen/patient focusing on the prevention and reduction of risk factors for T2DM. Via health literacy, individuals and families must improve their skills to notice signs of T2DM and motivate the person to visit a GP; this part of the meso level work and health literacy is a key tool. It also shows that policy interventions mostly on macro level have the potential for a broad population impact via diffusion throughout three levels.

Population-level interventions can be delivered via a range of approaches. Each is established by a policy measure 
and involves a tool to achieve change in risk exposure. On the other hand, regulations e.g. of fat content in food or sugary drink taxation are an important tool to macro level belonging to stuctural interventions. The tools are diverse and include reformulation of foods or structural and environmental measures (e.g. new infrastructure for active commuting, such as walking and bicycle lanes). Public awareness campaigns are to prevent or reduce risk factors and provide the target group with information necessary to influence health behaviour (Whittemore et al., 2016).

Such approaches have been advocated by WHO, which calls for multi-sectoral action that simultaneously addresses different sectors contributing to prevention of risk factors for T2DM while, at the same time, shaping an environment that facilitates and promotes adequate levels of physical activity (World Health Organization, 2016). In particular, policies hold potential in relation to their ability to expand upon individual effects to influence entire populations. In this way, the healthy public policies may offer one of the most efficient methods of improving and protecting public health. Policy interventions should be based on scientific knowledge and introduced at all levels of the conceptual framework to be effective (Brownson, Chriqui, \& Stamatakis, 2009).

Using only one part of the system is insufficient to obtain and maintain overall health and wellbeing. To improve chronic disease prevention and successfully influence diabetes prevention, management and clinical outcomes, the application of a multilevel approach is needed. The linkages between the healthcare system and the broader environment play an important role in the management of chronic illness to promote health and prevent chronic diseases such as diabetes. A multilevel approach can be designed to guide the diabetes prevention and management Moreover, it can be applied to a variety of chronic illnesses with appropriate adaptation.

To our knowledge, this is the first attempt to develop a framework for the evaluation of a chronic disease (with T2DM as an example) using the multilevel approach. This is the most important strength of the presented study. Lack of available literature is the most important limitation of the presented work; there is no published literature on the topic from Denmark. The study is based on international literature review only, and at the time of conduct, there was a limited available literature on the subject. An interview process with relevant stakeholders would make our results more relevant.

\section{Conclusion}

A conceptual framework is developed for the analysis of chronic disease prevention work according to the principles of a multilevel approach using T2DM as an example. The framework shows different levels/actors in the health care systems, which are theoretically in charge of risk factors influencing the disease in Denmark. Working at multiple levels (intrapersonal, interpersonal and structural level) and within multiple settings (medical settings, communities/municipalities, regions and government) plays an important role in shaping the environment/communities. There is a strong need to develop models for chronic diseases in which the multilevel approach is well integrated and therefore contributes to better population health. In the next step of the presented research, we applied the developed conceptual framework to analyse the multilevel work in selected municipalities in the Region of Southern Denmark. Results will be presented in the near future.

\section{Competing Interests Statement}

The authors declare that there are no competing or potential conflicts of interest.

\section{References}

Ansari, R. M., Dixon, J. B., \& Browning, C. J. (2013). Socio-Ecological Approach to Self-Management of Type 2 Diabetes: Physical Activity and Dietary Intervention. In Type 2 Diabetes. InTech.

Brownson, R. C., Chriqui, J. F., \& Stamatakis, K. A. (2009). Understanding evidence-based public health policy. American journal of public health, 99(9), 1576-1583. https://doi.org/10.2105/AJPH.2008.156224

Carstensen, B., Kristensen, J. K., Ottosen, P., Borch-Johnsen, K., \& Steering Group of the National Diabetes Register. (2008). The Danish National Diabetes Register: trends in incidence, prevalence and mortality. Diabetologia, 51(12), 2187-2196. https://doi.org/10.1007/s00125-008-1156-z

Cook, J. E., Purdie-Vaughns, V., Meyer, I. H., \& Busch, J. T. A. (2014). Intervening within and across levels: A multilevel approach to stigma and public health. Social Science \& Medicine (1982), 103, 101-109. https://doi.org/10.1016/j.socscimed.2013.09.023

de Montigny, J. G., Desjardins, S., \& Bouchard, L. (2017). The fundamentals of cross-sector collaboration for social change to promote population health. Global Health Promotion, O(April), 175797591771403. https://doi.org/10.1177/1757975917714036 
Deshpande, A. D., Dodson, E. A., Gorman, I., \& Brownson, R. C. (2008). Physical activity and diabetes: opportunities for prevention through policy. Physical therapy, 88(11), 1425-1435.

Fitzgerald, T. M., Williams, P. A., Dodge, J. A., Quinn, M., Heminger, C. L., Moultrie, R., ... \& Lewis, M. A. (2017). Program implementation approaches to build and sustain health care coordination for type 2 diabetes. Health promotion practice, 18(2), 306-313. https://doi.org/10.1177/1524839916643705

Golden, S. D., McLeroy, K. R., Green, L. W., Earp, J. A. L., \& Lieberman, L. D. (2015). Upending the social ecological model to guide health promotion efforts toward policy and environmental change. https://doi.org/10.1177/1090198115575098

Green, L. W., Brancati, F. L., Albright, A., \& Primary Prevention of Diabetes Working Group. (2012). Primary prevention of type 2 diabetes: integrative public health and primary care opportunities, challenges and strategies. Family practice, 29(suppl_1), i13-i23. https://doi.org/10.1093/fampra/cmr126

Guariguata, L., Whiting, D. R., Hambleton, I., \& Beagley, J. (2013). Global estimates of diabetes prevalence for 2013 and projections for 2035. Diabetes Research and Clinical Practice, 103(2), 137-149. https://doi.org/10.1016/j.diabres.2013.11.002

Health in all policies (HiAP) framework for country action. (2014). Health Promotion International, 29(January), i19-i28. https://doi.org/10.1093/heapro/dau035

Lindström, J., Wikström, K., Maggini, M., Icks, A., Kuske, S., Rothe, U., ... \& Zaletel, J. (2015). Quality indicators for diabetes prevention programs in health care targeted at people at high risk. Annali dell'Istituto superiore di sanita, 51, 187-191. http://doi.org/10.4415/ANN

LeRouge, C., Gaynor, M., Li, C. C., \& Ma, J. (2010, January). A multi-level technical infrastructure for diabetes chronic care management in china. In System Sciences (HICSS), 2010 43rd Hawaii International Conference on (pp. 1-10). IEEE.

Lewis, M. A., Fitzgerald, T. M., Zulkiewicz, B., Peinado, S., \& Williams, P. A. (2017). Identifying Synergies in Multilevel Interventions: The Convergence Strategy. Health Education \& Behavior, 44(2), 236-244.. https://doi.org/10.1177/1090198116673994

National Institute of Healthcare and Excellence. (2011). T ype 2 diabetes pre prev vention: population and community-le vel interv interventions entions, (May).

Paquette-Warren, J., Hayward, M. N., Tompkins, J. W., \& Harris, S. B. (2014). Time to evaluate diabetes and guide health research and policy innovation: The Diabetes Evaluation Framework (DEFINE). The Canadian Journal of Program Evaluation, 29(2), 1. https://doi.org/10.3138/cjpe.29.2.1

Paquette-Warren, J., Tyler, M., Fournie, M., \& Harris, S. B. (2017). The Diabetes Evaluation Framework for Innovative National Evaluations (DEFINE): Construct and Content Validation Using a Modified Delphi Method. Canadian Journal of Diabetes. https://doi.org/10.1016/j.jcjd.2016.10.011

Rutter, H., Savona, N., Glonti, K., Bibby, J., Cummins, S., Finegood, D. T., ... White, M. (2017). The need for a complex systems model of evidence for public health. The Lancet, 6736(17), 9-11. https://doi.org/10.1016/S0140-6736(17)31267-9

Salway, S., \& Green, J. (2017). Towards a critical complex systems approach to public health. Critical Public Health, 27(5), 523-524. https://doi.org/10.1080/09581596.2017.1368249

Schwarz, P. E. H., Schwarz, J., Schuppenies, A., Stefan, R., \& Schulze, J. (2016). Association of Schools of Public Health, Sage Publications, Inc., 122(2), 258-263.

Sørensen, M., Korsmo-Haugen, H. K., Maggini, M., Kuske, S., Icks, A., Rothe, U., ... \& Zaletel, J. (2015). Health promotion interventions in type 2 diabetes. Annali dell'Istituto superiore di sanita, 51(3), 192-198. https://doi.org/10.4415/ANN

Whitehead, M., Dahlgren, G., \& Gilson, L. (2001). Developing the policy response to inequities in health: a global perspective. Challenging inequities in health: From ethics to action, 309, 323. https://doi.org/10.1093/acprof:oso/9780195137408.003.0021

Whittemore, R., Melkus, G. D. E., Grey, M., Journal, S., Nursing, H., Summer, N., ... Melkus, G. D. E. (2016). Applying the Social Ecological Theory to Type 2 Diabetes Prevention and Management Applying the Social Ecological Theory to Type 2 Diabetes. Prevention and Management, 21(2), 87-99.

World Health Organization. (2016). Global report on diabetes. World Health Organization. 
Zaletel, J., Piletic, M., Lindström, J., Icks, A., Rothe, U., Sørensen, M., \& Maggini, M. (2015). National Diabetes Plans: can they support changes in health care systems to strengthen diabetes prevention and care?. Annali dell'Istituto superiore di sanita, 51, 206-208. http://doi.org/10.4415/ANN

\section{Copyrights}

Copyright for this article is retained by the author(s), with first publication rights granted to the journal.

This is an open-access article distributed under the terms and conditions of the Creative Commons Attribution license (http://creativecommons.org/licenses/by/4.0/). 